\title{
Suppression of pyruvate dehydrogenase kinase-2 re-sensitizes paclitaxel-resistant human lung cancer cells to paclitaxel
}

\author{
Hong Sun ${ }^{1}$, Anyou Zhu' ${ }^{1}$, Xiang Zhou ${ }^{2}$ and Fengchao Wang ${ }^{1}$ \\ ${ }^{1}$ Department of Clinical Laboratory Science, The First Affiliated Hospital of Bengbu Medical College, Bengbu, China \\ ${ }^{2}$ Department of Nuclear Medicine, Ren Ji Hospital, School of Medicine, Shanghai Jiao Tong University, Shanghai, China \\ Correspondence to: Fengchao Wang, email: wfc123489@sina.com \\ Xiang Zhou, email: zhouxiang1103@126.com \\ Keywords: glycolysis, pyruvate dehydrogenase kinase-2, drug resistance, NSCLC \\ Received: October 07, $2016 \quad$ Accepted: January 24, $2017 \quad$ Published: April 10, 2017 \\ Copyright: Sun et al. This is an open-access article distributed under the terms of the Creative Commons Attribution License 3.0 \\ (CC BY 3.0), which permits unrestricted use, distribution, and reproduction in any medium, provided the original author and source \\ are credited.
}

\section{ABSTRACT}

Despite impressive initial clinical responses, the majority of lung cancer patients treated with paclitaxel eventually develop resistance to the drug. Pyruvate dehydrogenase kinase-2 (PDK2) is a key regulator of glycolysis and oxidative phosphorylation, and its expression is increased in a variety of tumors. In this study, the role of PDK2 in mediating paclitaxel resistance in lung cancer cells was investigated using biochemical and isotopic tracing methods. Increased expression of PDK2 was observed in paclitaxel-resistant cells ascompared totheir parental cells. Down-regulation of PDK2 usingsiRNA increased the sensitivity to paclitaxel of resistant lung cancer cells. Targeting paclitaxel-resistant cells throughPDK2 knockdown was associated with reduced glycolysis rather than increased oxidative phosphorylation (OXPHOS). Moreover, combining paclitaxel withthe specific PDK2 inhibitor dichloroacetate had a synergistic inhibitory effect on the viability of paclitaxelresistant lung cancer cells. These results indicate that paclitaxel-induced expression of PDK2 serves as an important mechanism for acquired paclitaxel resistance of lung cancer cells. They also highlight the importance of PDK2 for potential therapeutic interventions in patients who have developed a resistance to paclitaxel.

\section{INTRODUCTION}

Non-smallcell lung cancer (NSCLC) is one of the most common malignant tumors and a leading cause of mortality worldwide. Chemotherapy is a crucial strategy for advancedstage NSCLC. Paclitaxel (Taxol), which targets microtubules of cancer cells, has been widely used in cancertreatment $[1,2]$. Paclitaxel disrupts the dynamic equilibrium between soluble tubulin dimers and their polymerized form to stabilize the microtubule structure. In addition, paclitaxel is an effective inhibitor of chromosomal replication by obstructing cancer cells in the late G2 or mitotic phases [3]. However, the efficiency of paclitaxel-based chemotherapy is limited by the development of acquired resistance. Increasedexpression of multidrug resistant proteins and anti-apoptotic proteins isthe main cause of paclitaxel resistance [4]. However, the specific molecular mechanisms involved in paclitaxel resistance are complex and not completely understood.

In 1920s, Otto Warburg demonstrated that cancer cells exhibit increased glycolysis, even when oxygen is abundant. This phenomenon of enhanced aerobic glycolysis is known as the Warburg effect $[5,6]$. Cancer cells, unlike normal cells, often use aerobic glycolysis instead of mitochondrial oxidative phosphorylation (OXPHOS). The Warburg effect is closely associated with drug resistance in cancer cells. Agents that target glycolysis or OXPHOS have shown promising efficacy in overcoming dug resistance [7-9].

Pyruvate dehydrogenase kinase (PDK) is one of the key regulators of glycolysis and oxidative phosphorylation. PDK phosphorylates pyruvate dehydrogenase (PDH) to inhibit the conversion of pyruvate to acetyl-CoA, and plays a key role in oxidative phosphorylation, proliferation, and 
tumor maintenance of cancer cells [10-18]. However, the correlation between PDK expression and paclitaxel resistance of cancer cells is still unclear. In this study, we investigated the molecular mechanisms involved in the paclitaxel-resistance of NSCLC cells, and the relationship betweenPDK2 and paclitaxel resistance. Our data show that paclitaxel-resistant NSCLC cellsexhibit increased expression of the PDK isoform 2 (PDK2) compared with their parental cells. Suppression of PDK2 or use of the PDK inhibitor dichloroacetate (DCA) increase sensitivity of NSCLC cells to paclitaxel. Importantly, combination of paclitaxel and DCA hasa synergistic inhibitory effect on theviabilityof NSCLC cells. Together, our results indicate that PDK2 plays an important role in paclitaxel resistance of NSCLC cells, and therefore serves as a promising therapeutic target for overcoming paclitaxel resistance in NSCLC.

\section{RESULTS}

\section{Selection and characterization of paclitaxel- resistant A549-R cells}

A549 cells were treated with increasing concentrations of paclitaxel in culture medium for selection of paclitaxel-

A

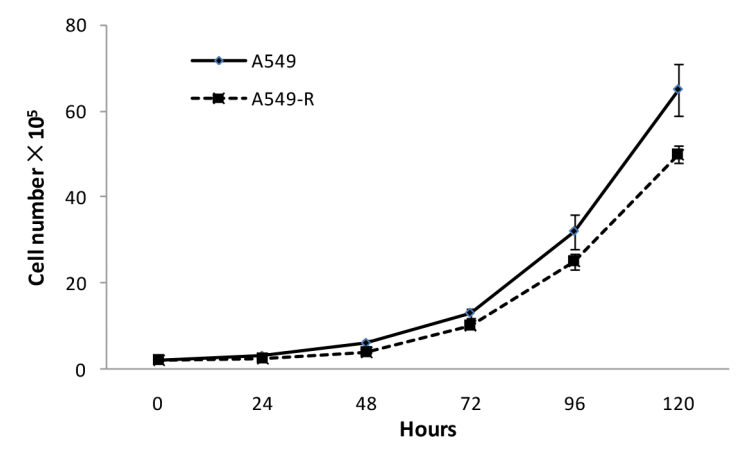

C

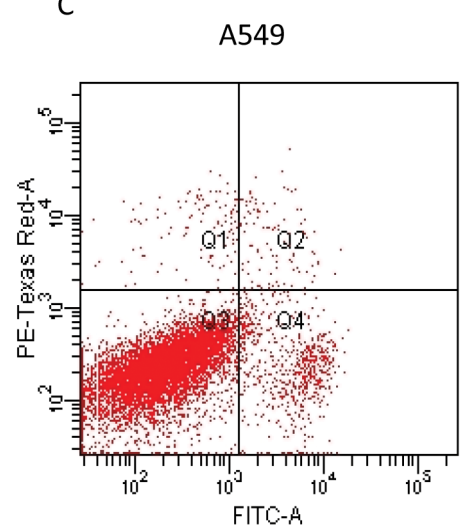

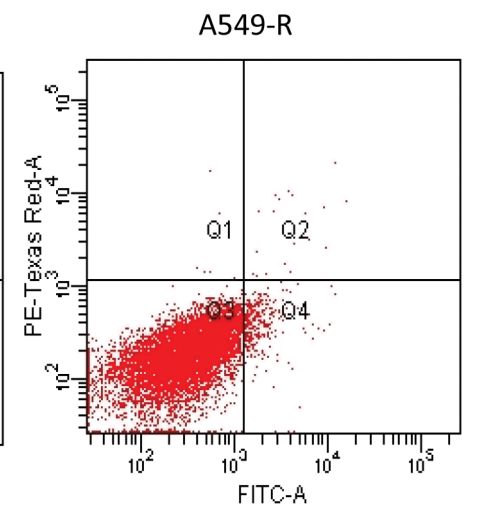

resistant cells. After 6 months, one resistant cell clone (A549-R) was obtained from the A549 parental cell line and was used for subsequent experiments.

As shown in Figure 1A, A549-R cells displayed a decreased growth rate compared with A549 cells. The CCK8 assay was performed to confirm the resistance to paclitaxel. The $\mathrm{IC}_{50}$ of paclitaxel was $142 \pm 9.5 \mathrm{nM}$ in A549-R cells and $16 \pm 2.8 \mathrm{nM}$ in A549 cells (Figure 1B). To compare the survival capacity of A549-R and A549 cells, the number of apoptotic cells was measured by flow cytometry. A549-R cells displayed a decreased apoptosis compared with A549 cellsincubated $24 \mathrm{~h}$ with $15 \mathrm{nM}$ paclitaxel (Figure 1C, 1D).

Glucose glycolysis and mitochondrial function in A549 and A549-R cells were assessed. A549-R cells showed a significant increase in glucose uptake (Figure 2A) and lactate production (Figure 2B) compared with A549 cells. Oxygen consumption rate (OCR) is linked to respiration and can be used as a surrogate marker for mitochondrial function. To estimate the mitochondrial function, the OCR was measured using glucose or glutamine as the carbon source. As shown in Figure 2C, A549-R cells displayed significantly reduced oxidative ability in the presence of glucose or glutamine as a carbon source.
B

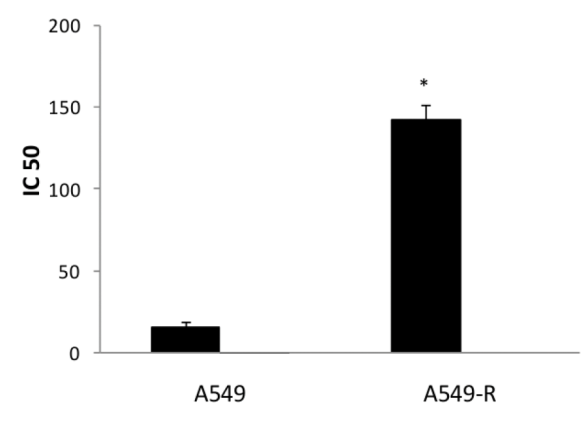

D

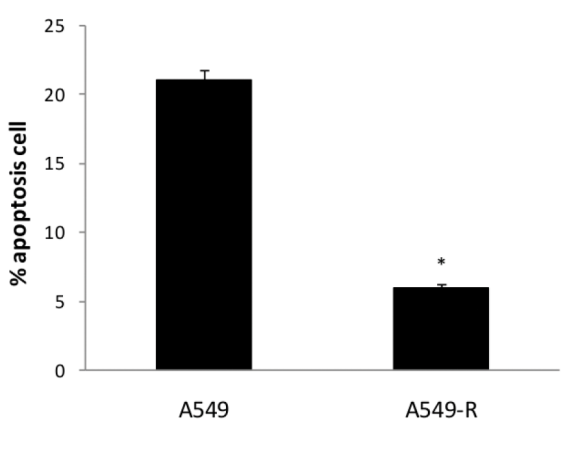

Figure 1: Characterization of paclitaxel-resistant A549-R cells. (A) Viability of A549 and A549-R cells determined by cell counting. (B) $\mathrm{IC}_{50}$ for paclitaxel in A549 and A549-R cells after paclitaxel treatment for $48 \mathrm{~h}$. (C and D) Apoptosis in A549 and A549-R cells treated with $10 \mathrm{nM}$ paclitaxel for $48 \mathrm{~h}$ by flow cytometry using Annexin V/PI staining and its representation in bar format. (*, P<0.05). 


\section{Inhibition of PDK2 restores A549-R cell sensitivity to paclitaxel}

The above results suggest that the paclitaxel resistance in A549 cells is associated with increased glycolysis and suppressed oxidative phosphorylation. As PDK2 is one of the key regulators of glycolysis and oxidative phosphorylation, its expression was examined in A549-T cells (A549 cells treated with $4 \mathrm{nM}$ paclitaxel for $48 \mathrm{~h}$ ), A549-R and A549-R1 cells (A549-R and A549-R1 cells have different paclitaxel resistance), and A549 cells(treated with $0 \mathrm{nM}$ paclitaxel) to evaluate the role of PDK2 in mediating paclitaxel resistance. PDK2 mRNA and protein levelswere markedly increased in A549-R cells compared to A549 cells (Figure 3A-3D). In addition, paclitaxel increased PDK2 expression in a dose-dependent manner in A549 cells (Figure 3B, 3C). These results indicate that PDK2 plays animportant role in paclitaxel resistance. Therefore, we investigated the effect of PDK2 suppression on paclitaxel sensitivity. As the expression of PDK2 was increased in A549-R and A549-R1 cells, we hypothesized that the down-regulation of PDK2 by siRNA
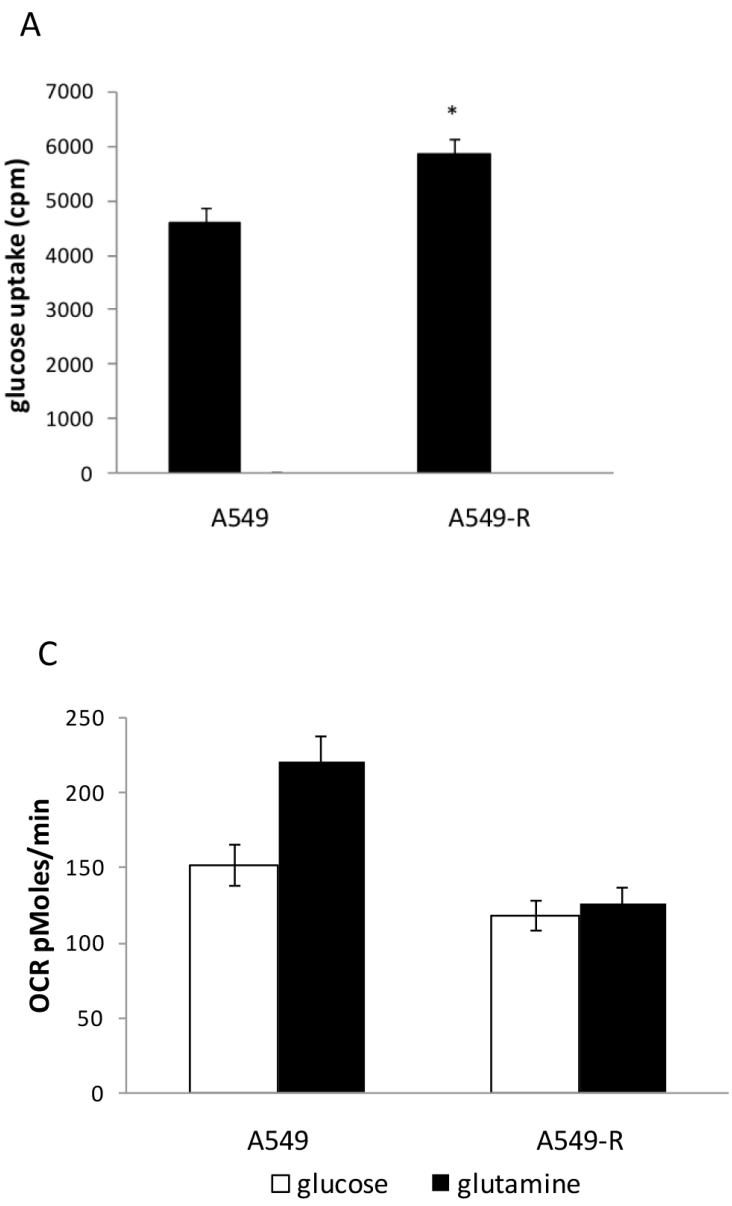

might re-sensitize A549-R cells to paclitaxel. To this end, PDK2 was suppressed with siRNA in A549-R and A549R1cells (Figure 3D), and the cells were then treated with different concentrations of paclitaxel. As shown in Figure $3 \mathrm{E}$, cell survival ratewasdecreasedin A549, A549-R, and A549-R1 cells with suppressed PDK2. Down-regulation of PDK2 inhibited survival rate of A549-R cellsmore than the less resistantA549-R1 cells and their parental A549 cells. A549-R cells showed increased sensitivity to paclitaxel compared to A549-R1 and their parental A549 cells(Figure 3F). These results indicate that paclitaxel resistance is associated with increased PDK2 expression and that knockdown of PDK2 may re-sensitize A549-R andA549-R1cells to paclitaxel.

\section{Targeting A549-R cells by PDK2 suppression is attributed to inhibited glycolysis rather than increased OXPHOS}

We further assessed whether suppression of PDK2 re-sensitizes A549-R cells to paclitaxel by suppression of glycolysis or activation of OXPHOS. As shown in

B

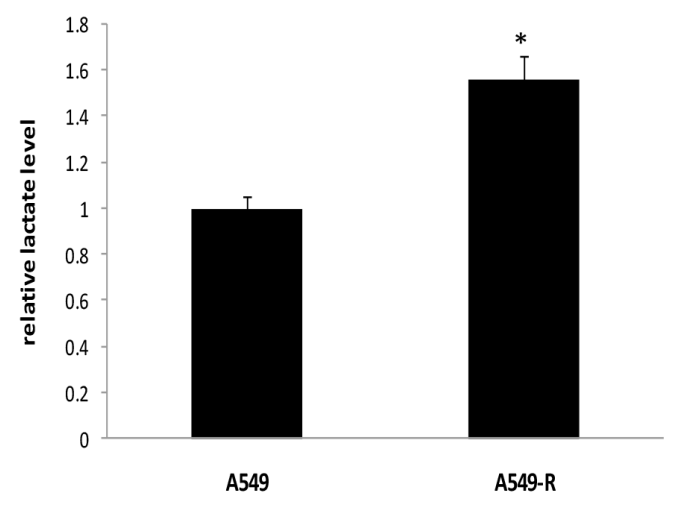

Figure 2: Changes in glycolysis in paclitaxel-resistant cells. (A) Comparison of glucose uptake in A549 and A549-R cells. (B) Lactate production rate detected in A549 and A549-R cells. (C) OCR in A549 and A549-R cells using glucose or glutamine as the carbon source. $(*, \mathrm{P}<0.05)$. 
Figure 4A, knockdown of PDK2 increased OCR by $10 \pm 3.8 \%$ in A549-R cells, and $28.5 \pm 5.5 \%$ in A549 cells $(P<0.01$, compared with untreated control). When glutamine was used as a carbon source, PDK2 siRNA inhibited glutamine oxidation through an unclear mechanism (Figure 4B). Furthermore, knockdown of PDK2 inhibited glutamine oxidation more effectively in drug resistant cells.

The Warburg effect is closely associated with drug resistance in cancer cells $[17,18]$. Glucose uptake and lactate generation ability areimportant indicatorsof cell glycolytic activity. In our study, we found that knockdown of PDK2 reduced glucose uptake and lactate production inA549-R cells (Figure 4C, 4D). Next, we analyzed the effect of PDK2 suppressionon ATP production and glycolysis. PDK2 suppression decreased ATP generation in A549-R cells, but failed to reduce ATP production in A549 cells (Figure 4E). These results indicate that targeting A549-R cells by PDK2 knockdown is attributed to inhibited glycolysis rather than increased OXPHOS in paclitaxel-resistant cells. To elucidate the mechanism of reduced glucose uptake and lactate production, the effect of PDK2 suppressionon the expression of glucose transporter1 (GLUT1) and the glycolytic enzyme lactate dehydrogenase A (LDHA) was investigated (Figure 5A).
A significant decrease in GLUT1 expression was observed following PDK2 knockdown in A549 and A549-R cells; however, expression of LDHA was not changed after PDK2 knockdown in A549 cells or A549R cells.

Next, we examined the effect of PDK2 knockdown on the expression of c-Myc and HIF-1 $\alpha$, two major transcription factors that regulate the expression of GLUT1. Interestingly, c-Myc protein levels were not changed after PDK2knockdown in A549 cells, but PDK2 suppression reduced the c-Myc levels compared to untreated control in A549-R. HIF-1 $\alpha$ levels were decreased after treatment in A549 and A549-R cells. In addition, PDK2 suppression decreased HIF-1 $\alpha$ in A549-R cells compared to A549 cells. These results indicate that PDK2suppression inhibits glycolysis more significantly in paclitaxel resistant cells.

\section{Combination of paclitaxel with dichloroacetate(DCA) shows a synergistic inhibitory effect on A549 cells}

DCA, a small chemical compound, inhibits PDKmediated inactivation of PDH (pyruvate dehydrogenase) and diverts glucose metabolism from glycolysis towards OXPHOS [13]. As shown in Figure 6A, the effect of
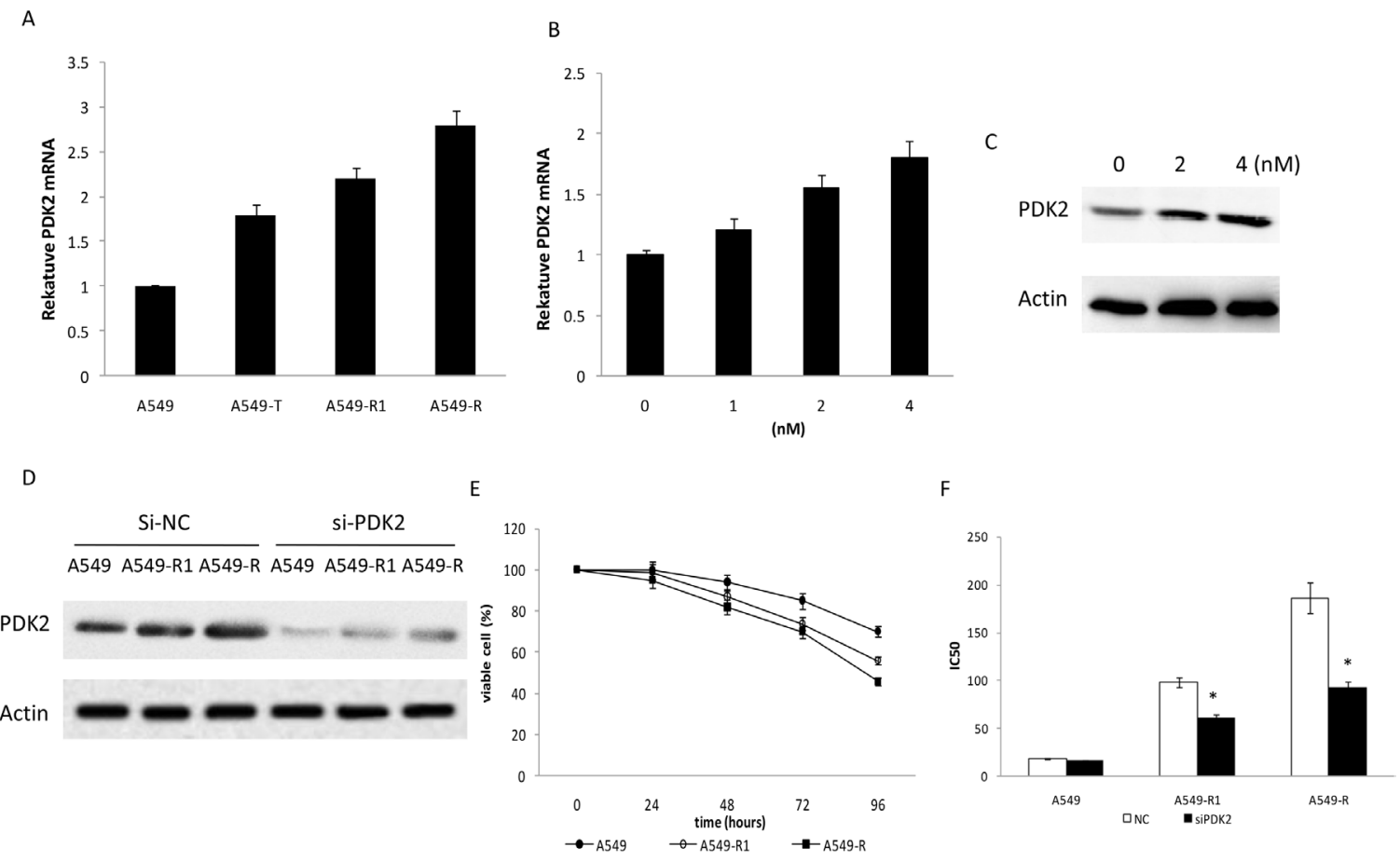

Figure 3: Increased PDK2 expression in paclitaxel-resistant cells. (A) Comparative analysis of mRNA levels of PDK2 in A549, A549-T, A549-R1 and A549-R cells. (B) PDK2 mRNA levels detected by qRT-PCR under increasing concentrations of paclitaxel in A549 cells. (C) Western blot performed with antibodies against PDK2 and actin on cell lysates from A549 cells treated with increasing concentrations of paclitaxel for 48 h. (D) Western blot performed with antibodies against PDK2 on cell lysates from A549, A549-R1 and A549-R cells transfected with scrambled siRNA (NC) or PDK2 siRNA. (E) Cell survival rate determined by cell counting after treatment

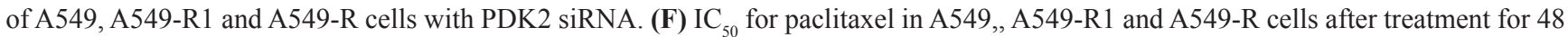
$\mathrm{h}$ with paclitaxel. Inhibition of PDK2 restored A549-R cell sensitivity to paclitaxel. (*, P<0.05). 
DCA on A549 cell viability was assessed. DCA treatment decreased cell viability in a dose-dependent manner in both A549 and A549-R cells. A549-R cells displayed increased sensitivity to DCA compared to A549 cells, consistent with the results of PDK2 knockdown by siRNA.

As down-regulation of PDK2 by siRNA or DCA inhibited the viability of A549-R cells, the effect of combining paclitaxel with DCA on paclitaxel-resistant cells was investigated. In A549-R cells, paclitaxel combined with DCA was markedly more effective in inhibiting cell viability compared with either agent used alone (Figure 6B). These results demonstrate that the combination of paclitaxel with DCA has a greater capacity to decrease the viability of paclitaxel-resistant cells compared to either agent used alone.
A

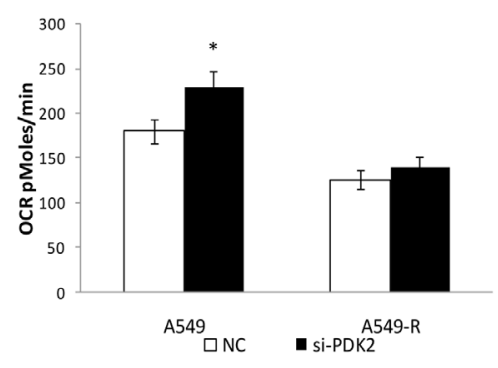

B

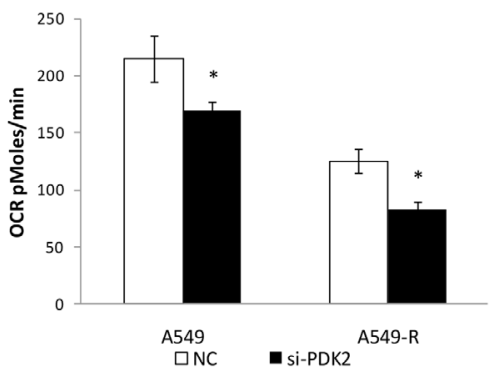

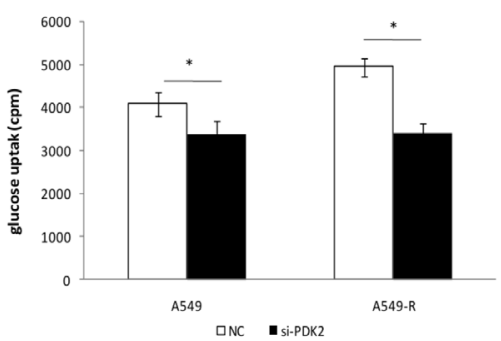

D

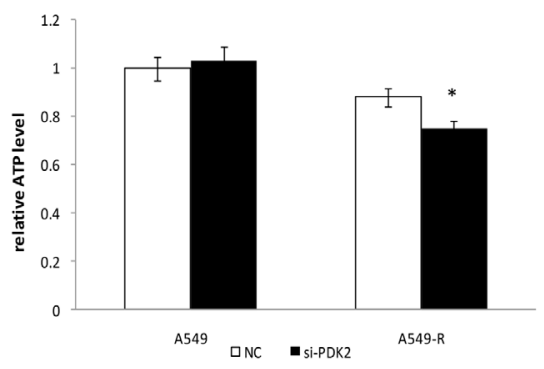

$E$

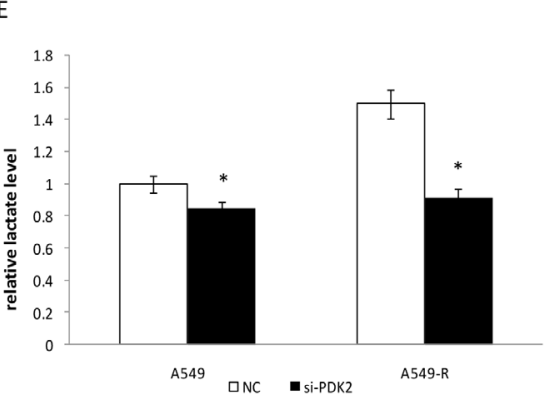

Figure 4: Inhibition of glycolysis in A549 and A549-R cells by PDK2 RNAi. (A) OCR in A549 and A549-R cells treated by siRNA-PDK2 for $48 \mathrm{~h}$ using glucose as the carbon source. (B) OCR in A549 and A549-R cells treated by siRNA-PDK2 for $48 \mathrm{~h}$ using glutamine as the carbon source. (C) Glucose uptake in A549 and A549-R cells transfected with PDK2 siRNA. Cells were incubated in DMEM with $18 \mathrm{~F}-\mathrm{FDG}$ for $1 \mathrm{~h}$ and radioactivity was measured using the well $\gamma$-counter. (D) Quantification of ATP levels in cells $48 \mathrm{~h}$ posttransfection with PDK2-siRNA. (E) Lactate production rate determined in A549 andA549-R cells 48 h post-transfection with PDK-siRNA. $(*, \mathrm{P}<0.05)$.

A
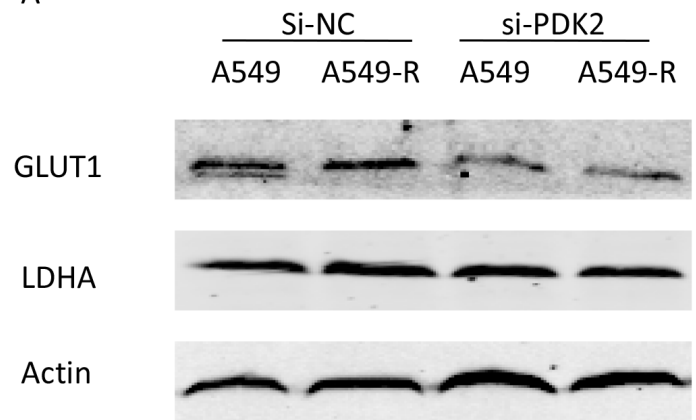

B

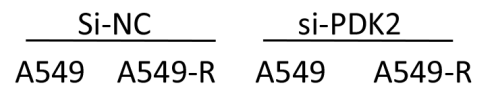

c-Myc

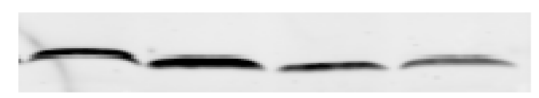

HIF-1 $\alpha$

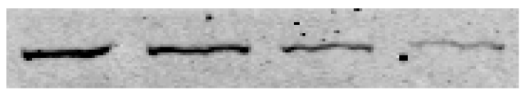

Actin

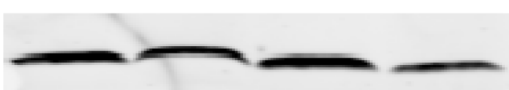

Figure 5: Protein expression changes post-transfection with PDK2-siRNA. (A) Effects of PDK2-siRNA on the expression of GLUT1 and LDHA $48 \mathrm{~h}$ post-transfection. (B) Effects of PDK2-siRNA on the expression of c-Myc and HIF-1 $\alpha 48 \mathrm{~h}$ post-transfection. (*, $\mathrm{P}<0.05$ ). 


\section{DISCUSSION}

In this study, the role of PDK2 in acquired paclitaxel resistance in A549 cells was investigated. In comparison to paclitaxel-sensitive cells, PDK2 expression was increased in paclitaxel-resistant cells. Knockdown of PDK2 increased sensitivity of paclitaxel-resistant cells to paclitaxel. Compared to paclitaxel-sensitive cells, A549-R cells displayed higher sensitivity to the PDK inhibitor DCA. In addition, when compared to single agent therapy, treating cells with the combination of paclitaxel and DCA exhibited an increased inhibitory effect on A549-R cells.

Paclitaxel is a widely used chemotherapeutic agent for the treatment of malignant tumors, including lung cancer. However, the resistance of cancer cells to paclitaxel is an important issue and can lead to subsequent recurrence and metastasis of malignant tumors [19-21]. To date, the specific molecular mechanisms involved in paclitaxel resistance are still poorly understood. Although the Warburg effect is closely associated with drug resistance in cancer cells, the relationship between the Warburg effect and paclitaxel resistance is unclear.

Previous studies have demonstrated that PDK2 plays a critical role in OXPHOS, glycolysis, and tumor maintenance of cancer cells [22, 23]. Suppression of PDK2 increases apoptosis of cancer cells via activated OXPHOS, suggesting that it might be used as a therapeutic strategy for treatment of malignant tumors. However, the role of PDK2 in paclitaxel resistance of cancer cells has not been studied. Our study indicates that paclitaxel increases PDK2 expression in A549 cells by increasing PDK2 transcription. Suppression of PDK2 by PDK2 siRNA or the inhibitor DCA increases sensitivity of A549 and A549-R cellsto paclitaxel. This indicates that the paclitaxel-induced PDK2 expression in paclitaxelresistant cells may be an adaptive mechanism in these cells to modulate glycolysis and OXPHOS to avoid the paclitaxel-inducedapoptosis. In addition, since PDK inhibition was more effective in suppressing cell growth of the resistant A549-R cells than the parental A549 cells,

A

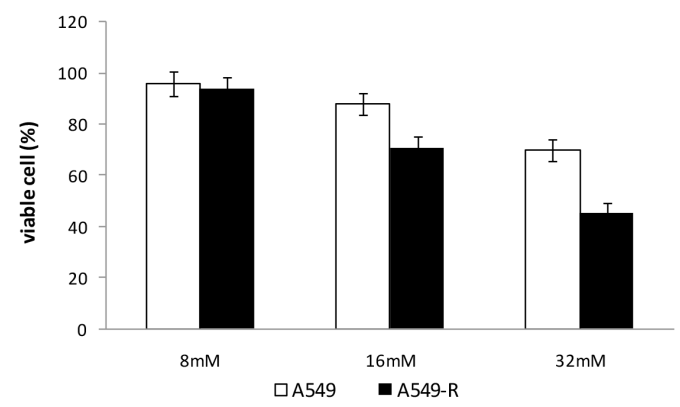

these results suggest that PDK2 may serve as an effective target for overcoming paclitaxel resistance in patients with lung cancer. To our knowledge, this is the first report to demonstrate the role ofPDK2 in acquired paclitaxel resistance in human lung cancer cells.

Previous studies have indicatedthat the Warburg effect contributes to drug resistance of cancer cells [24]. However, the underlying molecular mechanisms remain largely unclear. The current study demonstrates that the paclitaxelresistance of A549 cells is associatedwith increased glycolysis and suppressed oxidative phosphorylation. Furthermore, our study indicates that PDK2 suppression reversesthe paclitaxel resistance through suppression of glucose glycolysis rather than activation of OXPHOS in paclitaxel resistant cells.

Since inhibition of PDK2 decreased glucose uptake and lactate production, we investigated the effect ofPDK2 knockdown on the glycolytic enzyme LDHA and the glycolytic transporter GLUT1. A significant decrease in GLUT1 expression was observed after PDK2 knockdown in A549 cells. PDK2 knockdown therefore increased glucose uptake and lactate generation, possibly by upregulation of GLUT1 expression.

HIF-1 $\alpha$ and c-Myc are two major oncogenic transcription factors known to regulate metabolism in cancer cells [25-27]. HIF-1 $\alpha$ and c-Myc can up-regulate transcription of GLUT1, sustaining the Warburg effect in cancer cells $[28,29]$. PDK2 knockdown had no significant effect on c-Myc levels in A549 cells, but strongly inhibited c-Myc expression in paclitaxel resistant 549-Rcells. In addition, in both A549 cells and A549-R cells, PDK2siRNA decreased HIF- $1 \alpha$ levels. Since the effect of PDK2 knockdown was more pronounced in drug resistant cells, these results suggest that PDK2 can target paclitaxel resistant cells to inhibit glycolysis. The combination of paclitaxel with DCA was more effective in killing paclitaxel-resistant cells, compared to either paclitaxel or DCA treatment alone. The synergistic inhibitory effect of the combination therapy indicates that it might represent an effective strategy to overcome the paclitaxel resistance of NSCLC cells.

B

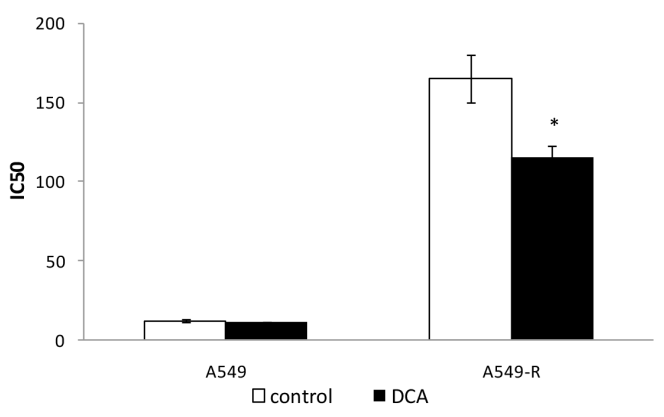

Figure 6: DCA resensitizes paclitaxel-resistant cells to paclitaxel. (A) Viability of A549 and A549-R cells treated with various concentrations of DCA for $48 \mathrm{~h}$. (B) $\mathrm{IC}_{50}$ for paclitaxel in A549 and A549-R cells after treatment for $48 \mathrm{~h}$ with 0 or $10 \mathrm{mM} \mathrm{DCA}$. (*, $\mathrm{P}<0.05$ ). 
In summary, the present study shows that PDK2 plays an important role in paclitaxel resistance, with paclitaxel-induced expression of PDK2 serving as an important mechanism for the acquired resistance of human lung cancer cells to paclitaxel. The results of this study provide valuable information for the development of targeted therapies for paclitaxel-resistanceby inhibiting PDK2, and highlight the importance of PDK2 in paclitaxel resistance.

\section{MATERIALS AND METHODS}

\section{Cell culture and transient transfection}

Human lung adenocarcinoma cell line A549 was obtained from the Chinese Academy of Sciences. A549R and A549R1 cells are paclitaxel-resistant clones developed as previously described [19]. Briefly, the parental A549 cellswere exposed to increasing paclitaxel concentrations, and the surviving resistant A549-R cells were cultured in DMEM containing $5 \mathrm{mM}$ glucose supplemented with $10 \%$ fetal bovine serum (FBS), sodium pyruvate and L-glutamine. A549 cells treated with $4 \mathrm{nM}$ paclitaxel for $48 \mathrm{~h}$ were designated as A549-T cells. Lipo2000 (Invitrogen) was used for transient transfection according to the manufacturer's protocol. Control siRNAand siRNAPDK2 were purchased from Pharma (China).

\section{Uptake of 18F-flurodeoxyglucose}

Cells were cultured in 12-well culture plates, then detached, washed twice, and subsequently incubated in $500 \mu \mathrm{l}$ of DMEM containing $4 \mu \mathrm{Ci} / \mathrm{mL}$ of ${ }^{18} \mathrm{~F}$-flurodeoxyglucose (18F-FDG) for $1 \mathrm{~h}$ at $37^{\circ} \mathrm{C}$. Pellets were washed twice with ice-cold phosphate buffered saline (PBS). Cell lysates were obtained using $500 \mu \mathrm{l}$ of $0.1 \mathrm{M}$ $\mathrm{NaOH}$, and then the radioactivity of the whole-cell lysates was assayed using a well $\gamma$-counter. The readouts were normalized to corresponding protein amounts (Beyotime).

\section{Lactate production assay}

Cells were seeded onto 6-well plates and transfected with PDK2 siRNA, or treated with DCA. Approximately $48 \mathrm{~h}$ after transfection, cells were washed and cultured in serum-free DMEM for $16 \mathrm{~h}$. Lactate levels in the medium were measured using a lactate assay kit (CMA Microdialysis). The readouts were normalized to corresponding protein amounts (Beyotime).

\section{Western blot analysis}

Cells were harvested and lysed in buffer containing $50 \mathrm{mM}$ Tris- $\mathrm{HCl}, 150 \mathrm{mM} \mathrm{NaCl}, 2 \mathrm{mM}$ ethylenediaminetetraacetic acid, 1\% Triton and $1 \mathrm{mM}$ phenylmethylsulfonyl-fluoride (PMSF; Sigma) for 10 min on ice. Protein concentration was determined by the bicinchoninic acid protein assay. Proteins were separated using sodium dodecylsulfate polyacrylamide gel electrophoresis, and transferred onto nitrocellulose membranes. The membranes were incubated $3 \mathrm{~h}$ at $37^{\circ} \mathrm{C}$ with primary antibodies in PBS with 5\% nonfat milk. The following primary antibodies were used: anti-PDK-2 rabbit antibody (1:1000; Abcam) and anti- $\beta$-actin antibody (1:2000, Sigma). Membranes were extensively washed with PBS and incubated with secondary anti-rabbit antibody (1:10000; LI-COR Biosciences). After washing with PBS, protein bands were visualizedand analyzed using a Gel DocXR system (Bio-Rad) and IMAGE LABTM software (version 2.0; Bio-Rad).

\section{Oxygen consumption rate analysis}

Oxygen consumption rate (OCR) was measured using the Seahorse XF24 analyzer (Seahorse Bioscience). Cells were seeded in a 24-well culture plate and allowed to attach overnight. Approximately $30 \mathrm{~min}$ prior to the assay, culture medium was changed to the Seahorse assay medium (DMEM containing $5 \mathrm{mM}$ glucose or $2 \mathrm{mM}$ L-glutamine) and OCR was measured according to the manufacturer's instructions.

\section{Cell survival rate CCK-8 assay}

Cell survival rate was examined using the Cell Counting Kit-8 (CCK-8; Dojindo Molecular Technologies), which is based on dehydrogenase activity detection in viable cells. Cells were plated in 96-well culture plates at a density of 6000 cells/well. After $24 \mathrm{~h}$, the medium was replaced with DMEM containing 10\% FBS and drugs at the indicated concentrations. Following $24 \mathrm{~h}$ incubation, $10 \mu \mathrm{L}$ of CCK-8 solution was added to each well, and cells were further incubated for $2 \mathrm{~h}$. Absorbance was read at $450 \mathrm{~nm}$ using a micro-plate reader. Tocalculate IC50, A549 and A549-Rcells werecultured with increasing paclitaxelconcentrations, and the $\mathrm{IC}_{50}$ value was calculated using the GraphPad Prism version 5.0 software.

\section{Cellular ATP level assay}

Relative cellular ATP content was measured using the ATP bioluminescent somatic cell assay kit (Sigma, St. Louis, MO, USA) according to the manufacturer's instructions. Briefly, $2 \times 10^{5}$ cells were seeded in a $24-$ well plate. After $24 \mathrm{~h}$, cells were washed, centrifuged, and lysed. Lysates were collected, and luminescence was measured using a luminescence reader and normalized to the protein concentration. Measurements were performed in triplicates [9]. 


\section{Statistical analysis}

Statistical differences between the groups were assessed using two-tailed ANOVA and t-tests. P values $<0.05$ were considered significant.

\section{Abbreviations}

DCA: dichloroacetate; GLUT1:glucose transporter 1; LDHA: lactate dehydrogenase A; NSCLC: Nonsmall-cell lung cancer; OCR: Oxygen consumption rate; OXPHOS: oxidative phosphorylation; PDH: pyruvate dehydrogenase; PDK2: Pyruvate dehydrogenase kinase-2.

\section{ACKNOWLEDGMENTS}

We thank Dr. Yaer Hu of Shanghai Jiao Tong University School of Medicine for her assistance in isotopic tracing analysis.

\section{CONFLICTS OF INTEREST} interests.

The authors declare that they have no competing

\section{FUNDING}

This work was supported by a grant from the National Natural Science Foundation of China (No. 81571710) and Bengbu medical college of science and technology development fund project (BYKF1405).

\section{REFERENCES}

1. Henley D, Isbill M, Fernando R, Foster JS, Wimalasena J. Paclitaxel induced apoptosis in breast cancer cells requires cell cycle transit but not Cdc2 activity. Cancer Chemother Pharmacol. 2007; 59:235-249.

2. Hida K, Akiyama K, Ohga N, Maishi N, Hida Y. Tumour endothelial cells acquire drug resistance in a tumour microenvironment. J Biochem. 2013; 153:243-249.

3. Frankel A, Buckman R, Kerbel SR. Abrogation of Taxolinduced G2-M Arrest and Apoptosis in Human Ovarian Cancer CellsGrown as Multicellular Tumor Spheroids. Cancer Res. 1997; 57:2388-2293.

4. DonnenbergVS, Donnenberg DA. Multiple Drug Resistance in Cancer Revisited: The Cancer Stem Cell Hypothesis. J Clin Pharmacol. 2005; 45:872-877.

5. Warburg O. On the origin of cancer cells. Science. 1956; 123:309-314.

6. Elf SE, Chen J. Targeting glucose metabolism in patients with cancer. Cancer. 2014; 120:774-780.

7. Xu RH, Pelicano H, Zhou Y, Carew JS, Feng L, Bhalla $\mathrm{KN}$, Keating MJ, Huang P. Inhibition of glycolysis in cancer cells: A novel strategy to overcome drug resistance associated with mitochondrial respiratory defect and hypoxia. Cancer Research. 2005; 65:613-621.

8. Zhou X, Chen R, Xie W, Ni Y, Liu J, Huang G. Relationship between 18F-FDG accumulation and lactate dehydrogenase A expression in lung adenocarcinomas. The journal of nuclear medicine. 2014; 55:1766-1771.

9. Sun H, Zhu A, Zhang L, Zhang J, Zhong Z, Wang F. Knockdown of PKM2 Suppresses Tumor Growth and Invasion in Lung Adenocarcinoma. Int J Mol Sci. 2015; 16:24574-24587.

10. Sutendra G, Dromparis P, Kinnaird A, Stenson TH, Haromy A, Parker JM, McMurtry MS, Michelakis ED. Mitochondrial activation by inhibition of PDKII suppresses HIF1 signaling and angiogenesis in cancer. Oncogene. 2013; 32:1638-1650.

11. Jha MK, Suk K. Pyruvate dehydrogenase kinase as a potential therapeutic target for malignant gliomas. Brain Tumor Res Treat. 2013; 1:57-63.

12. Zhou X, Chen R, Yu Z, Li R, Li J, Huang G. Dichloroacetate restores drug sensitivity in paclitaxelresistant cells by inducing citric acid accumulation. Mol Cancer. 2015; 14:63.

13. Bonnet S, Archer SL, Allalunis-Turner J, Haromy A, Beaulieu C, Thompson R, Lee CT, Lopaschuk GD, Puttagunta L, Bonnet S, Harry G, Hashimoto K, Porter CJ, et al. A mitochondria- $\mathrm{K}+$ channel axis is suppressed in cancer and its normalizationpromotes apoptosis and inhibits cancer growth. Cancer Cell. 2007; 11:37-51.

14. Choi YW, Lim IK. Sensitization of metformin-cytotoxicity by dichloroacetate via reprogramming glucose metabolism in cancer cells. Cancer letter. 2014; 346:300-308.

15. Lu CW, Lin SC, Chien $\mathrm{CW}$, Lin SC, Lee CT. Overexpression of pyruvate dehydrogenase kinase 3 increases drug resistance and early recurrence in colon cancer. Am J Pathol. 2011; 179:1405-1414.

16. Lu CW, Lin SC, Chen KF, Lai YY, Tsai SJ. Induction of pyruvate dehydrogenase kinase-3 by hypoxia-inducible factor-1 promotes metabolic switch and drug resistance. $\mathrm{J}$ Biol Chem. 2008; 283:28106-28114.

17. Xu RH, Pelicano H, Zhou Y, Carew JS, Feng L, Bhalla KN, Keating MJ, Huang P. Inhibition of glycolysis in cancer cells: a novel strategy to overcome drug resistance associated with mitochondrial respiratory defect and hypoxia. Cancer Res. 2005; 65:613-621.

18. Zhou Y, Tozzi F, Chen J, Fan F, Xia L, Wang J, Gao G, Zhang A, Xia X, Brasher H, Widger W, Ellis LM, Weihua Z. Intracellular ATP levels are a pivotal determinant of chemoresistance in colon cancer cells. Cancer Res. 2012; 72:304-314.

19. Sun QL, Sha HF, Yang XH, Bao GL, Lu J, Xie YY. Comparative proteomic analysis of paclitaxel sensitive A549 lung adenocarcinoma cell line and its resistant 
counterpart A549-Taxol. J Cancer Res Clin. 2011; 137:521-532.

20. Chen LP, Cai SM, Fan JX, Li ZT. PEBA Regimen (Cisplatin, Etoposide, Bleomycin, and Adriamycin) in the Treatment of Drug-Resistant Choriocarcinoma. Gynecologic Oncology. 2002; 56:231-234.

21. Januchowski R, Zawierucha P, Andrzejewska M, Ruciński M, Zabel M. Microarray-based detection and expression analysis of $\mathrm{ABC}$ and SLC transporters in drug-resistant ovarian cancer cell lines. Biomed Pharmacother. 2013; 67:240-245.

22. Bowker-Kinley MM, Davis WI, Wu P, Harris RA, Popov $\mathrm{KM}$. Evidence for existence of tissue-specific regulation of the mammalian pyruvate dehydrogenase complex. Biochem J. 1998; 329:191-196.

23. Koukourakis MI, Giatromanolaki A, Sivridis E, Gatter KC, Harris AL; Angiogenesis Research Group. Pyruvate dehydrogenase and pyruvate dehydrogenase kinase expression in non-smallcell lung cancer and tumorassociated stroma. Neoplasia. 2005; 7:1-6.
24. Pelicano H, Martin DS, Xu RH, Huang P. Glycolysis inhibition for anticancer treatment.Oncogene. 2006; 5:4633-4646.

25. Semenza GL. Regulation of cancer cell metabolism by hypoxia-inducible factor 1. Semin Cancer Bio. 2009; 19:12-16.

26. Dang CV, O'Donnell KA, Zeller KI, Nguyen T, Osthus RC, $\mathrm{Li}$ Fe. The c-Myctarget gene network. Seminars in Cancer Biology. 2006; 16:253-264.

27. Wahlström T, Arsenian Henriksson M. Impact of MYC in regulation of tumor cell metabolism. Biochim Biophys Acta. 2015; 1849:563-569.

28. Bensinger SJ, Christofk HR. New aspects of the Warburg effect in cancer cell biology. Semin Cell Dev Biol. 2012; 23:352-361.

29. Denko NC. Hypoxia, HIF1 and glucose metabolism in the solid tumour. Nat Rev Cancer. 2008; 8:705-713. 